\title{
Percutaneous elimination of the left atrial appendage in quest for effective and safe prevention of stroke in patients with atrial fibrillation
}

\author{
Radosław Pracoń ${ }^{1}$, Marcin Demkow ${ }^{1}$, Adam Witkowski², Witold Rużyłło ${ }^{3}$ \\ ${ }^{1}$ Coronary and Structural Heart Diseases Department, Institute of Cardiology, Warsaw, Poland \\ ${ }^{2}$ Interventional Cardiology and Angiology, Institute of Cardiology, Warsaw, Poland \\ ${ }^{3}$ Institute of Cardiology, Warsaw, Poland
}

Postep Kardiol Inter 2014; 10, 2 (36): 71-74

DOI: 10.5114/pwki.2014.43508

Atrial fibrillation (Afib) has been declared epidemic on the rise in our aging population. It is estimated that roughly one quarter of now middle-aged men and women will develop Afib during their lifespan [1, 2]. The residual risk of stroke in anticoagulated patients as calculated by the $\mathrm{CHA}_{2} \mathrm{DS}_{2}-\mathrm{VASc}$ score ranges from $1.3 \%$ per year for a score of 1 to $15.2 \%$ per year for a score of 9 depending on the presence of clearly defined risk factors [3]. Cardioembolic strokes are associated with adverse prognosis as depicted by a high rate of serious disability and mortality. The adverse prognosis may be attributable to relatively large clots that obstruct significant parts of the cerebral circulation as well as the characteristics of the affected population, which is mostly elderly, fragile, and often burdened with concomitant diseases. Thus, the need for safe and effective measures of stroke prevention in Afib patients is beyond doubt.

There is a large body of evidence demonstrating that therapy with vitamin $\mathrm{K}$ antagonists (VKA) reduces the risk of stroke in Afib patients roughly by $64 \%$ and ischemic stroke by $67 \%$ [4]. Furthermore, as shown in a meta-analysis by Ruff et al. published in 2013 in The Lancet, high-dose novel oral anticoagulants (NOAC) are even more effective as compared to VKA in preventing ischemic strokes/systemic embolism with RR 0.81, $95 \% \mathrm{Cl} 0.73-0.91$, which translates to 147 patients that need to be treated with a NOAC instead of VKA to prevent one additional embolic event [5]. It is important to note, however, that low-dose NOAC prevented fewer ischemic strokes as compared to warfarin so only highdose NOAC are associated with better outcomes. Consequently, European guidelines recommend that every pa- tient with a $\mathrm{CHA}_{2} \mathrm{DS}_{2}$-VASc score of 1 or more (i.e. most of the Afib population) be treated with oral anticoagulants for stroke prevention with the preference of NOAC [6].

There are two well-known downsides to oral anticoagulation, namely bleeding and compliance.

The risk of bleeding on oral anticoagulants (OAC) as depicted by the HAS-BLED score ranges from less than $2 \%$ per year to more than $4 \%$ per year depending on the presence of a number of risk factors [7]. There are data showing that in patients with a HAS-BLED score above 3 the risk of hemorrhagic events exceeds that of thrombotic events [8]. Furthermore, in patients with unstable international normalized ratio (INR) values during treatment with VKA the risk of bleeding increases significantly and is accompanied by a drop in treatment effectiveness [9]. With NOAC, one does not need to adjust drug dosage to any biochemical parameters of coagulation. Furthermore, NOAC have been said to be safer than VKA in terms of bleeding complications. Indeed, as shown by Ruff's meta-analysis the rate of the most catastrophic complication, i.e. hemorrhagic stroke, is significantly reduced (RR 0.49, 95\% Cl 0.38-064) but with its relatively low incidence 219 patients need to be treated to prevent one such event [5]. In fact, NOAC do not reduce major bleeding as compared to VKA and the frequency of gastrointestinal bleeding is even increased (RR 1.25, 95\% Cl 1.01-1.55).

For oral anticoagulation to be effective, it needs to be applied regularly and long-term. There are several reasons for non-compliance. The most obvious ones include bleeding complications, problematic INR control in the case of VKA, costs in the case of NOAC, and still too low

Corresponding author:

Radosław Pracoń MD, PhD, Coronary and Structural Heart Diseases Department, Institute of Cardiology, 42 Alpejska St, 04-628 Warsaw,

Poland, phone: +48 504211 12, e-mail: radekpracon@yahoo.pl

Received: 9.06.2014, accepted: 9.06.2014. 
awareness among both patients and medical professionals. Even in such a restrictively controlled environment as that imposed by randomized clinical trials, after a mean 2 to 2.5 years of follow-up $16.6 \%$ to $25.3 \%$ of patients ceased to receive anticoagulation, with similar numbers in NOAC and warfarin arms. The $10 \%$ per year dropout rate is far too high to be acceptable. The dropout curve may become more level during follow-up of patients who continue to receive the drug after 2 years, but there are real life data showing that after VKA treatment is initiated only about $30 \%$ of patients who started the treatment are still anticoagulated after 6 years follow-up [10, 11].

To sum up, even with the most state-of-the art pharmacological methods stroke prevention in Afib patients remains a significant challenge for contemporary medicine.

The rationale for mechanical closure of the left atrial appendage (LAAC) as a method of cardioembolic stroke prevention in Afib patients has been derived from pathophysiological reasoning and clinical observations. Atrial fibrillation disturbs the physiology of atrial blood flow in its passage from the pulmonary circulation to the left ventricle, leading to blood swirling and stasis within the left atrium and especially within its appendage, creating conditions for blood clotting. Indeed, clinical observations show $91 \%$ of left atrial thrombi to be located in its appendage [12]. Hence, mechanically sealing off the appendage from the blood circulation was hypothesized to reduce the risk of clot formation and consequently incidence of ischemic stroke.

Given its location, irregular and highly variable shape, as well as fragile walls, development of suitable, effective, and safe technology to close the appendage as well as the procedure itself has not been straightforward. The first device for percutaneous LAAC was the PLAATO system [13]. The first LAAC procedures in Poland were carried out with the use of PLAATO in the Institute of Cardiology in Warsaw by W. Rużyłło and A. Witkowski in May 2004. Out of the first 6 procedures the left atrial appendage (LAA) was successfully occluded in 5 cases and one procedure was abandoned due to unusual LAA anatomy. There were no periprocedural complications and at mean 54 months follow-up there were no ischemic strokes and no intracranial or gastrointestinal bleeding [14]. Despite promising preliminary results the PLAATO system was withdrawn from the market in 2006 [15]. Initial experience with PLAATO paved the way to new designs that are CE marked and currently in clinical use in Europe, i.e. the WATCHMAN device and the Amplatzer Cardiac Plug (ACP) device. The WATCHMAN device is based on a self-expanding nitinol cage covered with polyethylene membrane and equipped with barbs anchoring the device in the LAA. The ACP device consists of three self-expanding nitinol parts: a distal lobe with hooks filling the proximal part of the LAA, a connecting waist, and a proximal disk covering the ostium of the LAA for better stability and compete sealing of the appendage. Both devices are delivered transvenously via transseptal puncture using dedicated delivery systems. After implantation of such a device within weeks it becomes covered by endothelium and thus completely separates the appendage from the circulating blood. Apart from the two CE marked devices there are other technologies under development. One of those technologies, the LARIAT suture device has undergone preliminary clinical testing with promising results [16]. The Lariat requires both transseptal and epicardial access for delivery of a suture that is tightened over the external side of the LAA ostium.

The only randomized data on LAAC come from the WATCHMAN device. In the PROTECT AF clinical randomized trial the device proved noninferior to warfarin in reducing the risk of stroke, systemic embolization, and cardiovascular death (RR 0.62, 95\% Cl 0.35-1.25). At the early phases of clinical experience with the device implantation procedural complications such as serious pericardial effusion (4.8\%), procedure-related stroke (1.1\%), and device embolization $(0.6 \%)$ were noted [17]. As discussed above, the procedure is technically demanding and a steep learning curve has been observed. Thus, the number of periprocedural complications consequently decreased along with increasing experience with device implantation during the PROTECT AF trial and decreased further in the Continuous Access Protocol, where the pericardial effusion rate dropped to $2.2 \%$, with no reported periprocedural strokes [18]. Similarly, the device implantation success rate increased from 90.1\% in the PROTECT AF to $95 \%$ in the Continuous Access Protocol. The safety of implantation and clinical efficacy of the device have been further corroborated in the PREVAIL trial, the results of which were made public before the American College of Cardiology Scientific Sessions in 2013 [19]. Thus, in its most recent vote an FDA advisory panel almost unanimously recommended the WATCHMAN device as a safe and effective method of stroke risk reduction in patients with Afib.

The design of the ACP device is based on broad clinical experience with other members of the Amplatzer family of cardiovascular plugs and occluders. The published data on safety and efficacy of the ACP device are based on prospective registries and case series. The largest early European prospective registry showed a high implantation success rate (96\%) while major complications occurred in $7 \%$ of patients, similarly to early experience with the WATCHMAN device [20]. Encouraging early experience has also been reported based on a Polish case series from the Institute of Cardiology in Warsaw and more recently from the Silesian Centre for Heart Diseases in Zabrze [21, 22].

In the recent European Society of Cardiology update to guidelines on atrial fibrillation, LAAC has been indicated for patients with high risk of stroke and contraindications for oral anticoagulation [23]. The recommendation 
is very cautious (IIb), based mainly on the only available clinical trial with safety concerns that have already been discussed herein. Interestingly, the PROTECT AF trial did not enroll patients with contraindications to OAC. The target populations that could derive significant benefit from LAAC remain to be defined. At this point we can only hypothesize that apart from those who cannot be treated with $\mathrm{OAC}$, patients with vascular disease requiring long-term antiplatelet therapy, patients who experience cardioembolic complications while on OAC, and patients with oncological problems or renal failure may also derive significant benefit from percutaneous LAA elimination.

Yet another unresolved issue is the most optimal therapy after the successful procedure. It remains to be tested which patient subgroups require long-term OAC despite LAAC and which are candidates for short-term dual antiplatelet or short-term OAC therapy and if lifelong therapy with low-dose aspirin would be necessary afterwards.

In the face of the rapidly growing number of patients at risk of cardioembolic stroke associated with atrial fibrillation as well as limited compliance and risk of bleeding complications with currently available pharmacological preventive methods, there is no doubt that alternative methods of stroke prevention in Afib patients are needed. Percutaneous closure of the left atrial appendage is out of its infancy period and into adolescence. WATCHMAN and ACP devices are already available for clinical use while other designs are in the pipeline. The currently available data look very promising and with the national reimbursement programs for LAAC which are now in effect also in Poland we have the opportunity to observe the safety and effectiveness of the procedure in our daily clinical practice and look forward to real life data from individual centers and national registries. A broader spectrum of available evidence will help define patient subgroups who benefit the most from the procedure as well as better tailor the optimal treatment regimens after device implantation.

\section{References}

1. Go AS, Hylek EM, Phillips KA, et al. Prevalence of diagnosed atrial fibrillation in adults. National implications for rhythm management and stroke prevention: the AnTicoagulation and risk factors in atrial fibrillation (ATRIA) study. JAMA 2001; 285: 2370-5.

2. Stewart S, Hart CL, Hole DJ, McMurray JJ. Population prevalence, incidence, and predictors of atrial fibrillation in the Renfrew/ Paisley study. Heart 2001; 86: 516-21.

3. Lip GY, Frison L, Halperin JL, Lane DA. Identifying patients at high risk for stroke despite anticoagulation: a comparison of contemporary stroke risk stratification schemes in an anticoagulated atrial fibrillation cohort. Stroke 2010; 41: 2731-8.

4. Hart RG, Pearce LA, Aguilar MI. Meta-analysis: antithrombotic therapy to prevent stroke in patients who have nonvalvular atrial fibrillation. Ann Intern Med 2007; 146: 857-67.
5. Ruff CT, Giugliano RP, Braunwald E, et al. Comparison of the efficacy and safety of new oral anticoagulants with warfarin in patients with atrial fibrillation: a meta-analysis of randomised trials. Lancet 2014; 383: 955-62.

6. European Heart Rhythm Association, European Association for Cardio-Thoracic Surgery; Camm AJ, Kirchhof P, Lip GY, et al. Guidelines for the management of atrial fibrillation: the task force for the management of atrial fibrillation of the European Society of Cardiology (ESC). Eur Heart J 2010; 31: 2369-429.

7. Pisters R, Lane DA, Nieuwlaat R, et al. A novel user-friendly score (HAS-BLED) to assess 1-year risk of major bleeding in patients with atrial fibrillation: the Euro Heart Survey. Chest 2010; 138: 1093-100.

8. Gallego P, Roldan V, Torregrosa JM, et al. Relation of the HASBLED bleeding risk score to major bleeding, cardiovascular events, and mortality in anticoagulated patients with atrial fibrillation. Circ Arrhythm Electrophysiol 2012; 5: 312-8.

9. Connolly SJ, Pogue J, Eikelboom J, et al. Benefit of oral anticoagulant over antiplatelet therapy in atrial fibrillation depends on the quality of international normalized ratio control achieved by centers and countries as measured by time in therapeutic range. Circulation 2008; 118: 2029-37.

10. Sudlow M, Thomson R, Thwaites B, et al. Prevalence of atrial fibrillation and eligibility for anticoagulants in the community. Lancet 1998; 352: 1167-71.

11. Partington SL, Abid S, Teo K, et al. Pre-admission warfarin use in patients with acute ischemic stroke and atrial fibrillation: the appropriate use and barriers to oral anticoagulant therapy. Thromb Res 2007; 120: 663-9.

12. Blackshear JL, Odell JA. Appendage obliteration to reduce stroke in cardiac surgical patients with atrial fibrillation. Ann Thorac Surg 1996; 61: 755-9.

13. Ostermayer SH, Reisman M, Kramer PH, et al. Percutaneous left atrial appendage transcatheter occlusion (PLAATO system) to prevent stroke in high-risk patients with non-rheumatic atrial fibrillation: results from the international multi-center feasibility trials. J Am Coll Cardiol 2005; 46: 9-14.

14. Kukula K, Klopotowski M, Konka M, et al. Left atrial appendage occlusion using the PLAATO system in high-risk patients with atrial fibrillation - long-term follow-up. Postep Kardiol Inter 2009; 5: 51-7.

15. Bayard YL, Omran H, Neuzil P, et al. PLAATO (percutaneous left atrial appendage transcatheter occlusion) for prevention of cardioembolic stroke in non-anticoagulation eligible atrial fibrillation patients: results from the european PLAATO study. Eurointervention 2010; 6: 220-6.

16. Bartus K, Han FT, Bednarek J, et al. Percutaneous left atrial appendage suture ligation using the LARIAT device in patients with atrial fibrillation: initial clinical experience. J Am Coll Cardiol 2013; 62: 108-18.

17. Reddy VY, Holmes D, Doshi SK, et al. Safety of percutaneous left atrial appendage closure: results from the watchman left atrial appendage system for embolic protection in patients with AF (PROTECT AF) clinical trial and the continued access registry. Circulation 2011; 123: 417-24.

18. Reddy VY, Doshi SK, Sievert H, et al. Percutaneous left atrial appendage closure for stroke prophylaxis in patients with atrial fibrillation: 2.3-year follow-up of the PROTECT AF (watchman left atrial appendage system for embolic protection in patients with atrial fibrillation) trial. Circulation 2013; 127: 720-9. 
19. Holmes DR. Final results of randomized trial of left atrial appendage closure versus warfarin for stroke/thromboembolic prevention in patients with non-nalvular atrial fibrillation (PREVAIL). released prior to: American College of Cardiology Scientific Session/i2 summit 2013.

20. Park JW, Bethencourt A, Sievert H, et al. Left atrial appendage closure with amplatzer cardiac plug in atrial fibrillation: initial european experience. Catheter Cardiovasc Interv 2011; 77: 700-6.

21. Demkow M, Witkowski A, Cedzynski L, et al. Transcatheter closure of left atrial appendage with the amplatzer cardiac plug in patients with atrial fibrillation and high risk of thromboembolic events: early experience. Kardiol Pol 2010; 68: 250-4.

22. Streb W, Szymala M, Kukulski T, et al. Percutaneous closure of the left atrial appendage using the amplatzer cardiac plug in patients with atrial fibrillation: evaluation of safety and feasibility. Kardiol Pol 2013; 71: 8-16.

23. Camm AJ, Lip GY, De Caterina R, et al. 2012 focused update of the ESC guidelines for the management of atrial fibrillation: an update of the 2010 ESC guidelines for the management of atrial fibrillation: developed with the special contribution of the European Heart Rhythm Association. Europace 2012; 14: 1385-413. 